\title{
EFFICACY OF LIVE ATTENUATED FOWL ADENOVIRUS SEROTYPE 8B ISOLATE OF MALAYSIA IN SPECIFIC PATHOGEN-FREE CHICKENS
}

\author{
NORFITRIAH MOHAMED SOHAIMI ${ }^{1,2^{*}}$ and MOHD HAIR BEJO ${ }^{1,2}$ \\ ${ }^{1}$ Department of Veterinary Laboratory Diagnostics, Faculty of Veterinary Medicine, \\ Universiti Putra Malaysia, 43400 Serdang, Selangor, Malaysia \\ ${ }^{2}$ Institute of Bioscience, Universiti Putra Malaysia, 43400 Serdang, Selangor, Malaysia \\ *E-mail: fitriahsohaimi@upm.edu.my
}

Accepted 13 December 2021, Published online 31 December 2021

\begin{abstract}
Fowl adenovirus (FAdV) is the primary agent of inclusion body hepatitis (IBH) in poultry and caused serious economic impact due to high mortality and poor productivity. To date, clinical cases of IBH in chickens increases over the years in Malaysia, thus, an effective local vaccine to control the disease outbreak is in dire need. The objective of the study was to determine the efficacy of live attenuated FAdV serotype 8b isolate, UPM1137CEL35, in specific-pathogen-free chickens. Attenuated isolate UPM1137CEL35 conferred full protection against the virulent FAdV isolate, UPM11134 in the vaccinated chickens. Neither clinical signs nor mortality were recorded in all vaccinated groups. However, in the challenged unvaccinated group, chickens showed clinical signs of weakness, reduced feed consumption, and lateral recumbency at day 4 to 7 postchallenge. Additionally, the body weight was low significantly $(p<0.05)$ compared to the challenged vaccinated groups. Upon necropsy, the liver from the challenged unvaccinated group was pale at peripheral bilateral lobes with the presence of focal lymphoid aggregation microscopically, while, challenged vaccinated groups were normal without significant changes. All vaccinated chickens were protected from disease manifestations with antibody response compared to the challenged unvaccinated group. It was concluded that the attenuated FAdV isolate, UPM1137CEL35, has a high potential to be used as a vaccine against the FAdV serotype $8 \mathrm{~b}$ of Malaysian isolates.
\end{abstract}

Key words: Attenuated, Fowl adenovirus (FAdV), inclusion body hepatitis (IBH), serotype 8b, vaccine

\section{INTRODUCTION}

Fowl adenovirus (FAdV) has been classified into Aviadenoviridae genus under a family of Adenoviridae and infects poultry species in cases of inclusion body hepatitis (IBH), hepatitishydropericardium syndrome (HHS), and gizzard erosion (Kajan et al., 2013; Zhang et al., 2016; Jordan et al., 2019; Mirzazadeh et al., 2021). According to the International Committee on Taxonomy of Viruses (ICTV) classification, FAdV comprised five molecular species designated as A to E and 12 serotypes (Benko et al., 2005).

FAdV infections are a major threat to the poultry industry with serious economic losses due to high mortality and poor productivity (Morshed et al., 2017; Norfitriah et al., 2018; Cui et al., 2020;

* To whom correspondence should be addressed.
Cizmecigil et al., 2020). FAdV serotype 2, 8a, 8b and 11 has been reported as primary agent of IBH (Kajan et al. 2013; Marek et al., 2016, Schachner et al., 2016) while HHS is mainly caused by FAdV serotype 4 (Yasmeen et al., 2017; Li et al. 2018; Rashid et al., 2020). Cases of gizzard erosion in chickens mainly due to FAdV serotype 1,8a, and 8b (DomanskaBlicharz et al., 2011; Grafl et al., 2012; Schachner et al., 2018; Morshed et al., 2017; Mirzazadeh et al., 2021).

Based on previous literature, vaccine development has been extensively conducted for FAdV $-4,8 b, 9$, and 11 due to the high prevalence rate in disease outbreaks compared to other serotypes worldwide (Matthews, 2005; Alvarado et al., 2007; Du et al., 2017; Wang et al., 2018; Steer-Cope et al., 2019). Vaccination is the most effective method for control and prevention against the disease either by horizontal or vertical transmission in poultry farms (Schijns et al., 2014; Schachner et al., 2018). 
Conventional vaccines such as the live attenuated type induce long-lasting immunity as early as day 7 post-vaccination (pv) and peak at day 21 pv by single-shot vaccination (Mansoor et al., 2011; Ali et al., 2015). Similarly, in cases of chickens infected with FAdV strain via the oral route, the highest antibodies titer was recorded at day 21 postinoculation (pi) (Maiti \& Sarkar, 1997). Both compartments of the immune system comprised of humoral and cellular mediated immunity were stimulated by the application of live attenuated vaccine in chickens (Schonewille et al., 2010; Ali et al., 2015). As compared to the inactivated vaccine, the level of antibody titer in the systemic circulation is shortened and declined rapidly at day $21 \mathrm{pv}$ (Mansoor et al., 2011).

Meanwhile, live attenuated vaccine was practically implemented in breeder flocks in Australia since 1989 from virulent serotype $8 \mathrm{~b}$ to protect broiler flocks via maternal antibodies from vaccinated parent flocks. Recently, the IBH outbreak was still reported in their broiler farms and involved various serotypes of FAdV which in this case, a cross-protection vaccine is required (Steer et al., 2011; Shah et al., 2017; Schachner et al., 2018; Kajan et al., 2019).

IBH was first reported in Malaysia in 2005 by Hair-Bejo, then the cases of IBH were kept increased over a year due to unavailable local vaccine against the serotype 8b in Malaysia (Juliana et al., 2014; Norina et al., 2016; Mat Isa et al., 2019). Although strict biosecurity and proper management were implemented in commercial premises, IBH was still reported in the country mainly in broiler and layer chickens (Norfitriah et al., 2018). Recently, FAdV serotype $8 \mathrm{~b}$ from Malaysian isolate was identified as a primary agent of IBH and highly pathogenic in chickens (Norfitriah et al., 2019). In the past few years, IBH was identified as a secondary disease and low pathogenic in chickens after being initiated by immunosuppressive diseases such as infectious bursal disease virus (IBDV) or chicken anemia virus (CAV). Although vaccination against IBDV and CAV were implemented in commercial premises, IBH cases remained high in Malaysia due to virulent FAdV strain from serotype $8 \mathrm{~b}$. Thus, it is an urgent need for a suitable local vaccine to control disease outbreaks in commercial poultry farms. The objective of this study was to determine the efficacy of attenuated FAdV serotype $8 \mathrm{~b}$ isolate in specific pathogen-free (SPF) chickens.

\section{MATERIALS AND METHODS}

\section{FAdV attenuated isolate}

FAdV vaccine inoculum was prepared from attenuated isolate in primary culture of chicken embryo liver (CEL) cells by serial passages (Sohaimi et al., 2019). The isolate was obtained from passage 35 namely as UPM1137CEL35 which was characterized as FAdV group E serotype 8b. Several molecular changes were detected in the major capsid proteins typically in hexon and fiber genes as described by Sohaimi et al. (2019). Freezing and thawing to aid the release of virus particles from the cell into supernatant were conducted three times before centrifugation at $216 \times g$ for $5 \mathrm{~min}$ at $4^{\circ} \mathrm{C}$ for clarification. Viral supernatant was titrated for tissue culture infective dose $50 \%$, $^{\text {TCID }}$ I0 $_{50}$ using primary CEL cells according to Reed and Muench (1938) protocol. The titer was determined at $10^{6.7}$ $\mathrm{TCID}_{50} / \mathrm{mL}$ and used as vaccine inoculum in this study.

\section{Preparation of Challenge Virus}

FAdV isolate named UPM11134 was originated from 18 days old broiler chickens in the IBH case with a history of daily mortality from day 7 of age. The mortality rate was 0.5 to $1.0 \%$ per day. Upon necropsy, the liver was swollen and pale with yellowish discoloration as well as hemorrhages and multifocal area of necrosis. The isolate was confirmed as FAdV by conventional PCR and was highly pathogenic in day-old specific pathogen-free (SPF) chickens with $100 \%$ mortality within 4 days pi with swollen and pale liver (Norfitriah et al., 2019). It was characterized as FAdV serotype 8 and was used as a challenge virus with the titer of $10^{8.3} \mathrm{TCID}_{50} / \mathrm{mL}$ based on Reed and Muench protocols (1938).

\section{Experimental Design}

The study was conducted under the approval of the Institutional Animal Care and Use Committee (IACUC), Universiti Putra Malaysia with AUP No. R076/2015. Forty-day-old SPF White Leghorns chicks were obtained from Thailand and divided into three major groups, namely groups A, B, and C (Table 1). The chicks in each group were housed separately in the experimental room at Animal Research Facility (ARF), Faculty of Veterinary Medicine, Universiti Putra Malaysia under controlled conditions at $25^{\circ} \mathrm{C}$ room temperature in the metal cage with wire mesh bedding. Each group of chicks was further divided into two groups namely the non-challenged and challenged groups. A total of 12 chicks in group A comprised eight chicks in the non-challenged group and labeled as A1 and four chicks in the challenged group labeled as A2. Four chickens were used for each period of sampling and made a total of 12 chicks per group (Sohaimi et al., 2019). All chicks in group A were vaccinated with $0.1 \mathrm{~mL}$ attenuated FAdV UPM1137CEL35 isolate with virus titer of $10^{6.7} \mathrm{TCID}_{50} / \mathrm{mL}$ at day old via the oral route. Similarly, twelves chicks in group B were divided into two groups namely the non-challenged (B1) and challenged (B2) groups. All chicks in group B 
Table 1. Experimental design for the efficacy of attenuated FAdV isolates in SPF chickens. The table shows a number of chicks given or not given vaccine by route of delivery and post-vaccination sampling schedule

\begin{tabular}{|c|c|c|c|c|}
\hline \multirow{2}{*}{ Groups } & \multicolumn{3}{|c|}{ Day post-vaccination (pv) } & \multirow{2}{*}{ Total chick per group } \\
\hline & 0 & 14 & 28 & \\
\hline \multicolumn{5}{|l|}{ Group A (Oral) } \\
\hline A1 (Non-challenged) & - & 4 & 4 & 12 \\
\hline A2 (Challenged) & - & - & 4 & \\
\hline \multicolumn{5}{|l|}{ Group B (Subcutaneous) } \\
\hline B1 (Non-challenged) & - & 4 & 4 & 12 \\
\hline B2 (Challenged) & - & - & 4 & \\
\hline \multicolumn{5}{|l|}{ Control (Unvaccinated) } \\
\hline C1 (Non-challenged) & 4 & 4 & 4 & 16 \\
\hline C2 (Challenged) & - & - & 4 & \\
\hline Total number of SPF chicks & & & 40 & \\
\hline
\end{tabular}

were vaccinated with $0.1 \mathrm{~mL}$ attenuated FAdV UPM1137CEL35 isolate with virus titer $10^{6.7} \mathrm{TCID}_{50} /$ $\mathrm{mL}$ at day old via subcutaneous (SQ) route. Sixteen chicks were assigned in group $\mathrm{C}$ with twelve chicks in the non-challenged group (C1) and four chicks in the challenged group (C2). All chicks in group $\mathrm{C}$ remained unvaccinated and acted as the control group throughout the trial. All chicks in challenged groups from groups A2, B2, and C2 were inoculated with $0.2 \mathrm{~mL}$ of pathogenic UPM11134 FAdV inoculum (Norfitriah et al., 2019) with a titer of $10^{8.3} \mathrm{TCID}_{50} /$ $\mathrm{mL}$ via intraperitoneal (IP) route at day 14 pi. All chicks were monitored daily for clinical signs and mortality and given ad libitum access to feed and water intake throughout the trial. The chicks were sacrificed at days 0,14 , and 28 post-vaccination (pv) in the control group (C1) and at days 14 and $28 \mathrm{pv}$ in both the groups $\mathrm{A} 1$ and $\mathrm{B} 1$ by cervical dislocation. The chicks in the challenged groups (A2, B2, \& C2) were sacrificed at day 14 post-challenge $(\mathrm{pc})$ or 28 days pv. Bodyweight was measured followed by blood collection for detection of FAdV antibody by ELISA test.

\section{Monitoring of clinical signs and mortality}

All chickens were monitored daily for mortality and clinical signs associated with FAdV infection such as depression, ruffled feather, diarrhea, inappetence, and weakness throughout the trial (Sohaimi et al., 2019).

\section{Gross lesion and histopathology}

Upon necropsy, the chicken carcasses were examined for gross lesions based on sampling days. Samples of trachea, liver, and gizzard were collected and fixed in $10 \%$ buffer formalin for histological examination using hematoxylin and eosin ( $\mathrm{H} \& \mathrm{E})$ staining (Bancroft \& Stevens, 1996).

\section{Enzyme-Linked Immunoabsorbent Assay}

The serum of chickens was tested for FAdV antibody using a commercial Enzyme-Linked Immunoabsorbent Assay (ELISA) kit (BioChek, UK). The test was conducted according to the manufacturer's protocol as described by previous work (Sohaimi et al., 2019).

\section{Statistical Analysis}

Means body weight and antibody titers in chickens were analyzed by IBM SPSS Statistics software version 22 using one-way analysis of variance (ANOVA). Before performing the ANOVA test, data normality was determined by the ShapiroWilk test. $\log 10$ transformation was applied for nonnormally distributed data. The significant difference between group means was determined with a value alpha level $p<0.05$ for all statistical tests. Multiple group comparison or post-hoc test was conducted after obtaining significant differences between group means. At the group level, Tukey HSD (Honest Significant Difference) test was conducted to compare between group means. For comparison between two groups means, data were evaluated by an independent t-test (Wilcox, 1990).

\section{RESULTS}

\section{Clinical signs}

None of the chickens vaccinated at day-old with FAdV attenuated isolate, UPM1137CEL35 (groups A1 \& B1) exhibits abnormal signs associated with FAdV throughout the trial. All chickens in groups A and B were active and alert as well as chickens in the control group until day $28 \mathrm{pv}$. After being challenged, chickens in the unvaccinated group (C2) showed clinical signs of weakness, severe depression, ruffled 
feathers, reduced feed consumption, and lateral recumbence started from day $4 \mathrm{pc}$ till $7 \mathrm{pc}$ before recovery at day $8 \mathrm{pc}$. Yet, mild depression, ruffled feathers, and reduced feed consumption were observed until day $14 \mathrm{pc}$ in group $\mathrm{C} 2$. All vaccinated challenged chickens in groups A2 and B2 were normal without showing any abnormal clinical signs associated with FAdV infection throughout the trial after the challenge.

\section{Bodyweight}

For vaccinated groups, there is no significant difference $(p>0.05)$ between non-challenged (A1 \& B1) and challenged (A2 \& B2) groups. The mean body weight of chickens in the control challenged group (C2) was significantly declined $(p<0.05)$ with $278 \pm 22 \mathrm{~g}$ at day $14 \mathrm{pc}$ compared to the nonchallenged (C1) group ( $423 \pm 40 \mathrm{~g})$. Among the nonchallenged groups (A1 \& B1), there is no significant difference $(p>0.05)$ in mean body weight until day 28 pv. In addition, there are statistically significant differences $(p<0.05)$ in mean body weight between challenged groups at day $14 \mathrm{pc}$. Mean body weight in the control group $(278 \pm 22 \mathrm{~g})$ was significantly low $(p<0.05)$ compared to that of vaccinated group A2 $(377 \pm 21 \mathrm{~g})$ and B2 $(379 \pm 19 \mathrm{~g})$ at day $14 \mathrm{pc}$ (Figure 1). In the control group (Group C), challenged chickens had low body weight significantly as compared to the non-challenged group $(p<0.05)$. Chicken in the challenged control group (group C2) was significantly lower in mean body weight at day $14 \mathrm{pc}(p<0.05)$ as compared to vaccinated groups with attenuated FAdV isolate, UPM1137CEL35 via oral (Group A2) and subcutaneous (Group B2) routes.

\section{Gross lesions}

For non-challenged chickens, liver, gizzard, and trachea in the control (C1) and vaccinated groups (A1 \& B1) were normal at days 0,14 , and 28 pv. On day $14 \mathrm{pc}$, the liver in chickens from the challenged and unvaccinated group (C2) was pale with yellowish discoloration at periphery bilateral lobes, while, gizzard and trachea were normal without significant findings in all control chickens (Figure 2a $\& 2 b)$. In vaccinated challenged groups (A2 \& B2), liver, gizzard, and trachea were normal at day $14 \mathrm{pc}$ without a significant finding.

\section{Histological lesions}

Liver, gizzard, and trachea were normal at days 0,14 , and $28 \mathrm{pv}$ in group C1. Similarly, for groups $\mathrm{A} 1$ and $\mathrm{B} 1$, there are no significant changes under microscopic examination at days 14 and $28 \mathrm{pv}$. In challenged groups (A2, B2, or C2), histological changes were observed in the liver with a presence of focal lymphoid aggregation and mild congestion in the control unvaccinated group (C2) at day $14 \mathrm{pc}$ (Figure $3 a$ and $3 b$ ).

\section{FAdV antibody response}

FAdV antibodies were not detected at day-old chicks. For the vaccinated group, A, the antibody titer in the challenged group, A2 $(5496 \pm 1688)$ was

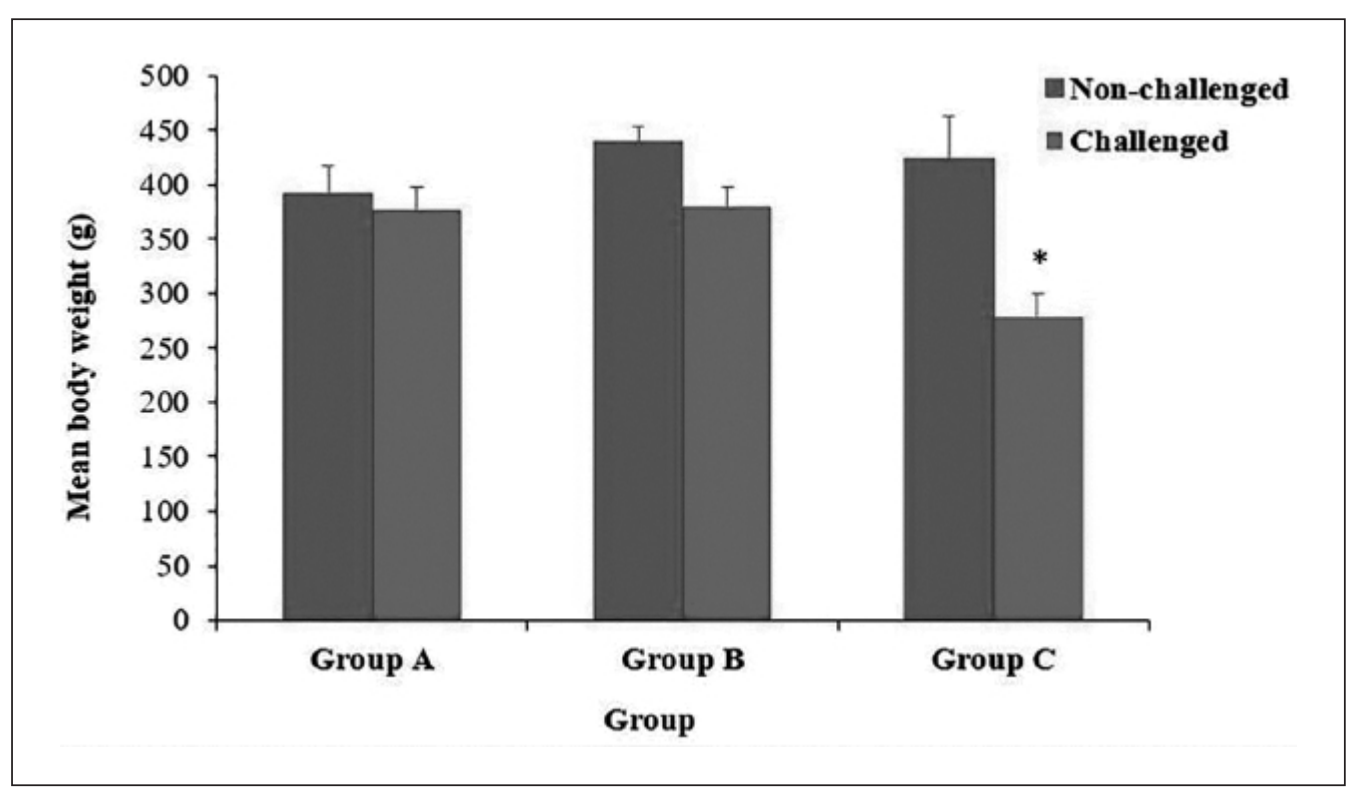

Fig. 1. Mean body weight of chickens in between non-challenged and challenged groups with virulent fowl adenovirus isolate, UPM11134, at day 14 post-challenged (pc). Both non-challenged and challenged groups consist of groups A, B, and C in the study. Group A: Vaccinated chickens with UPM1137CEL35 isolate via the oral route. Group B: Vaccinated chickens with UPM1137CEL35 isolate via the subcutaneous route. Group C: Control unvaccinated chickens. An asterisk * indicates a significantly different $(p<0.05)$ between groups. 


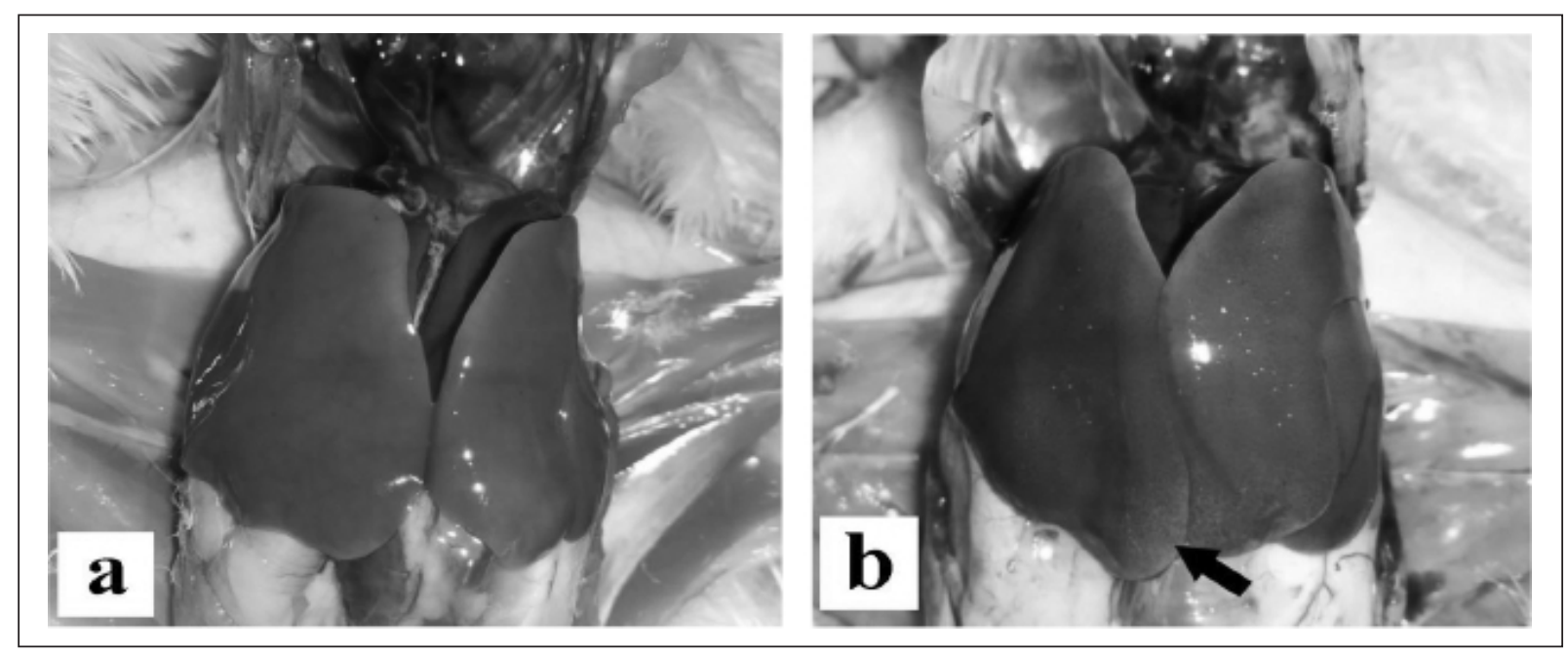

Fig. 2. Liver of chicken at day 14 pc after challenge with UPM11134 isolate from (a). Group B2 and (b). Group C2. Group B2: Vaccinated chicken with UPM1137CEL35 isolate via the subcutaneous route. Normal liver color (brown), smooth and glistening surface without the appearance of gross lesion was observed. Group C2: Control unvaccinated chicken. Gross lesion of the pale liver with yellowish discoloration in bilateral lobes was observed (arrow).

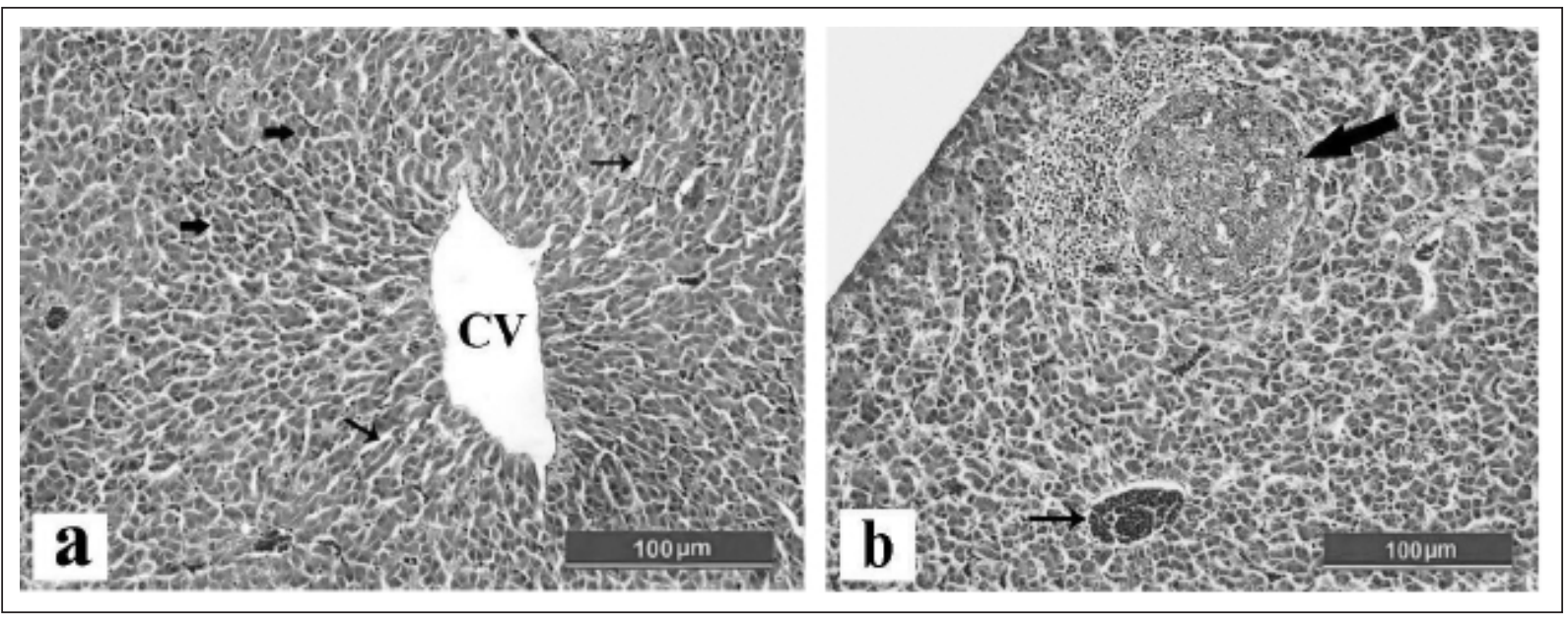

Fig. 3. Liver of chickens in challenged groups at day $14 \mathrm{pc}$ after challenged with UPM11134 isolate from (a). Group B2 and (b). Group C. Liver was normal in the inoculated group without changes in a central vein $(\mathrm{CV})$ and hepatocytes (thick arrows) lining by sinusoid (line arrows) in group B2. A histological lesion with focal lymphoid aggregation (thick arrow) with engorgement of red blood cells (RBCs) in a central vein (line arrow) indicates mild congestion at day 14 pc in Group C2. Group B2: Vaccinated chicken with UPM1137CEL35 via the subcutaneous route. Group C2: Control unvaccinated chicken. HE. Scale bar $=100 \mu \mathrm{m}$.

significantly high $(p<0.05)$ compared to the nonchallenged group, A1 (163 \pm 17$)$. For group B, there is no significant difference $(p>0.05)$ in antibody titer between challenged, B2 $(3895 \pm 1858)$, and nonchallenged group, B1 (324 \pm 85$)$. Analysis of antibody response in group $\mathrm{C}$ revealed the antibody titer in the challenged group, C2 $(6276 \pm 1983)$ had significantly high $(p<0.05)$ compared to that nonchallenged group, C1 (Not detected) (Figure 4). However, there is no significant different $(p>0.05)$ in mean antibody response between challenged groups. For non-challenged groups, the antibody titers in group B1 were significantly high $(p<0.05)$ compared to the control group $(\mathrm{C} 1)$ at days 14 and $28 \mathrm{pv}$. The antibody titers between-group A1 and B1 were not significantly different $(p>0.05)$ at days 14 and $28 \mathrm{pv}$.

\section{DISCUSSION}

The level of protection produced by the UPM1135CEL35 isolate against the challenge FAdV isolate, UPM11134 was evaluated based on clinical signs or mortality, bodyweight for 


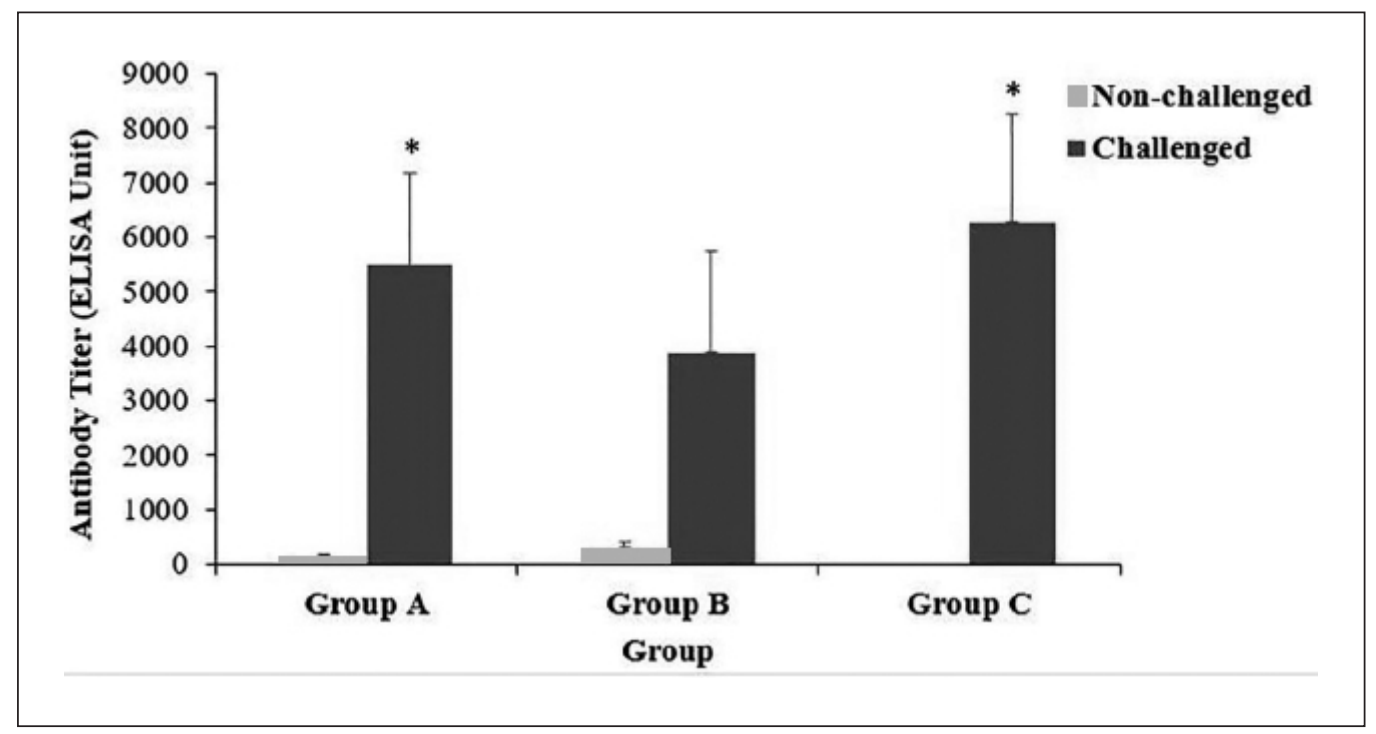

Fig. 4. Mean antibody titer of chickens between non-challenged and challenged groups at day 14 pc. Both non-challenged and challenged groups consist of groups A, B, and C in the study. Group A: Vaccinated chickens with UPM1137CEL35 isolate via the oral route. Group B: Vaccinated chickens with UPM1137CEL35 isolate via the subcutaneous route. Group C: Control unvaccinated chickens. An asterisk * indicates a significantly different $(p<0.05)$ between groups.

bird's performance, gross and histological lesions (Alvarado et al., 2007). It was demonstrated that FAdV attenuated serotype $8 \mathrm{~b}$ isolate, UPM1137CEL35 from cell culture-derived conferred full protection in vaccinated chickens from virulent FAdV serotype 8b isolate, UPM11134. Vaccinated chickens in both groups were safe and protected from disease without exhibiting clinical signs associated with IBH disease and lacked mortality throughout the trial. The challenged virus does not affect the overall performance of vaccinated chickens throughout the trial. This finding was consistent with previous studies using live attenuated FAdV isolate as a vaccine (Schonewille et al., 2010; Mansoor et al., 2011; Ali et al., 2015).

In contrast, chickens in the unvaccinated challenged group (C2) exhibited clinical signs of severe depression, ruffled feathers, and lateral recumbence at day 4 to $7 \mathrm{pc}$ before recovery at day 8 pc probably due to age resistance toward FAdV infection. All the chickens have ruffled feathers with reduced feed consumption until day $14 \mathrm{pc}$. In addition, significant low body weight was noted due to reduced feed consumption after exposure to virulent FAdV strain with no protection by vaccination. Those clinical signs are typically associated with IBH which is consistent with natural outbreaks and experimental induced studies in affected chickens (Junnu et al., 2015; Norina et al., 2016; Norfitriah et al., 2019; Wang et al., 2020).

Neither gross nor histological lesions were recorded in all tissues for vaccinated groups $\mathrm{A}$ and $B$ regardless of challenged with virulent FAdV strain.
As compared to the non-vaccinated and challenged group, C2, pale at bilateral lobes of the liver was observed at day $14 \mathrm{pc}$. This finding was identical with earlier observation conducted by Norfitriah et al. (2019) which the UPM11134 isolate caused yellowish and pale liver in infected chickens. Histologically, lymphoid aggregation with mild congestion was observed in the control group (C2) without the presence of intranuclear inclusion body (INIB) due to the recovery process as a result of virus replication in the liver at day $14 \mathrm{pc}$. In a study conducted by Cook (1974), chicken at an early age within 4 days old were highly susceptible to FAdV infection however it was completely resistant at 10 days of age.

High antibody response at day 14 pc occurs due to induction of active immune response caused by FAdV challenged virus. This observation was corroborated with the finding of Kaur et al. (1997). It is shown that chickens vaccinated by the SQ route had a slight low antibody titer due to progressive neutralization of antigen by existing antibody in the circulation and caused a slight reduction of antibody level at day $14 \mathrm{pc}$ following challenged with virulence FAdV. In contrast, chickens in the unvaccinated group (C2) had the highest antibody response than vaccinated groups due to a lack of FAdV antibodies to neutralize the antigen. Although all vaccinated chickens had low antibody response at day $14 \mathrm{pv}$ prior challenge, it seemed that chickens were protected against IBH due to the presence of adequate neutralizing antibodies or perhaps other acquired immune responses such as cell-mediated 
immunity. These findings are consistent with previous work using live FAdV serotype 4 vaccines which provide full protection in SPF chickens regardless of the absence of neutralizing antibodies (Schonewille et al., 2010).

FAdV is commonly spread by horizontal transmission via ingestion of infected feces in a commercial flock with contaminated litter (Li et al., 2017; Schachner et al., 2018). For this reason, the vaccination is crucial as early as day-old chicks by hatchery vaccination via a subcutaneous route which is more effective and convenient to poultry farmers (Abdul-Cader et al., 2018). In this way, all chicks will receive an equal amount of vaccine with high uniformity of FAdV antibody response and provide early protection in the flock. Based on the present findings, it seems that vaccinated chicks using attenuated FAdV isolate in primary CEL cells were able to induce protective immunity with a $100 \%$ survival rate in chickens after 2 weeks postchallenged without compromising the bird's performance. It indicates that the attenuated FAdV isolate, UPM1137CEL35 is safe and effective to be used as a vaccine candidate for Malaysian's poultry industry.

\section{CONCLUSION}

Live attenuated FAdV serotype $8 \mathrm{~b}$ isolate, UPM1137CEL35, confers full protection against the IBH in chickens. Thus, this is isolate has a high potential to be used as a homologous local serotype $8 \mathrm{~b}$ vaccine in Malaysian commercial poultry farms.

\section{ACKNOWLEDGEMENTS}

The study was funded by the Ministry of Education, Malaysia, and Ministry of Science and Technology, Malaysia with grant numbers 6369101 and 6364002, respectively.

\section{REFERENCES}

Abdul-Cader, M.S., Palomino-Tapia, V., Amarasinghe, A., Ahmed-Hassan, H., Senapathi, U.D.S. \& Abdul-Careem, M.F. 2018. Hatchery vaccination against poultry viral diseases: potential mechanisms and limitations. Viral Immunology, 31(1): 23-33.
Ali, S., Mahmood, M.S., Hussain, I. \& Khan, M.N. 2015. Preparation and evaluation of lyophilized live attenuated vaccine of inclusion body hepatitis hydropericardium syndrome (IBH-HPS) against challenge in broiler chickens. International Journal of Agriculture and Biology, 17(3): 658-662.

Alvarado, I.R., Villegas, P., El-Attrache, J., Jensen, E., Rosales, G., Perozo F. \& Purvis, L.B. 2007. Genetic characterization, pathogenicity, and protection studies with an avian adenovirus isolate associated with inclusion body hepatitis. Avian Diseases, 51: 27-32.

Bancroft, J.D. \& Stevens, A. 1996. Theory and Practice of Histological Technique. 5th Ed. Livingston Publishers, Edinburgh. 213-242 pp.

Benko, M., Harrach, B., Both, G.W., Russell, W.C., Adair, B.M., Adam, E., de Jong, J.C., Hess, M., Johnson, M. \& other authors. 2005. Family adenoviridae. In: Virus Taxonomy: Eighth Report of the International Committee on Taxonomy of Viruses. C.M. Fauquet, M.A. Mayo, J. Maniloff, U. Desselberger and L.A. Ball. (Eds.). Elsevier/Academic Press, London. pp. 213-228.

Cizmecigil, U.Y., Umar, S., Yilmaz, A., Bayraktar, E., Turan, N., Tali, B., Aydin, O., Tali, H.E., Yaramanoglu, M., Yilmaz, S. G., Kolukisa, A., Sadeyen, J.R., Iqbal, M. \& Yilmaz, H. 2020. Characterisation of Fowl Adenovirus (FAdV-8b) Strain Concerning the Geographic Analysis and Pathological Lesions Associated with Inclusion Body Hepatitis in Broiler Flocks in Turkey. Journal of Veterinary Research, 64(2): 231-237.

Cook, J.K.A. 1974. Pathogenicity of avian adenoviruses for day old chicks. Journal of Comparative Pathology, 84(4): 505-515.

Cui. J., Xu, Y., Zhou, Z., Xu, Q., Wang, J., Xiao, Y., Li, Z. \& Bi, D. 2020. Pathogenicity and molecular typing of Fowl Adenovirus-associated associated with hepatitis/hydropericardium syndrome in Central China (2015-2018). Frontiers in Veterinary Science, 7: 190.

Domanska-Blicharz, K., Tomczyk, G., Smietanka, K., Kozaczynski, W. \& Minta, Z. 2011. Molecular characterization of fowl adenoviruses isolated from chickens with gizzard erosions. Poultry Science, 90(5): 983-989.

Du, D., Zhang, P., Li, X., Tian, H., Cheng, Y., Sheng, D., Han, X., Shan, Y., Li, X., Yuan, Y., Zhang, H., Xue, J., Liu, W. \& Tian, K. 2017. Cell-culture derived fowl adenovirus serotype 4 inactivated vaccine provides complete protection for virus infection on SPF chickens. Virus Disease, 28(2): 182-188. 
Grafl, B., Aigner, F., Liebhart, D., Marek, A., Prokofieva, I., Bachmeier, J. \& Hess, M. 2012. Vertical transmission and clinical signs in broiler breeders and broilers experiencing adenoviral gizzard erosion. Avian Pathology, 41(6): 599-604.

Hair-Bejo M. 2005. Inclusion body hepatitis in a ûock of commercial broiler chickens. Jurnal Veterinar Malaysia, 7: 23-26.

Jordan, A.B., Blake, L., Bisnath, J., Ramgattie, C., Carrington, C.V. \& Oura, C.A.L. 2019. Identification of four serotypes of fowl adenovirus in clinically affected commercial poultry co infected with chicken infectious anaemia virus in Trinidad and Tobago. Transboundary and Emerging Diseases, 66(3): 1341-14348.

Junnu, S., Lertwatcharasarakul, P., Jala, S., Phattanakulanan, S., Monkong, A., Kulprasertsri, S., Thivalai, C., Chakritbudsabong, W., Chaichoun, K. \& Songserm, T. 2015. An inactivated vaccine for prevention and control of inclusion body hepatitis in broiler breeders. Thai Journal of Veterinary Medicine, 45(1): 5562.

Juliana, M.A., Nurulfiza, M.I., Hair-Bejo, M., Omar, A.R. \& Aini, I. 2014. Molecular characterization of fowl adenoviruses isolated from inclusion body hepatitis outbreaks in commercial broiler chickens in Malaysia. Pertanika Journal of Tropical Agriculture Science, 37(4): 483-497.

Kaján, G.L., Affranio, I., Tóthné Bistyák, A., Kecskeméti, S. \& Benkõ, M. 2019. An emerging new fowl adenovirus genotype. Heliyon, 5(5): e01732.

Kajan, G.L., Kecskeméti, S., Harrach, B. \& Benkõ, M. 2013. Molecular typing of fowl adenoviruses, isolated in Hungary recently, reveals high diversity. Veterinary Microbiology, 167: $357-$ 363.

Kaur, A., Oberoi, M.S. \& Singh, A. 1997. Neutralising antibody and challenge response to live and inactivated avian adenovirus-1 in broilers. Tropical Animal Health and Production, 29: 141-146.

Li, P.H., Zheng, P.P., Zhang, T.F., Wen, G.Y., Shao, H.B. \& Luo, Q.P. 2017. Fowl adenovirus serotype 4: Epidemiology, pathogenesis, diagnostic detection, and vaccine strategies. Poultry Science, 96(8): 2630-2640.

Maiti, N.K. \& Sarkar, P. 1997. Humoral immune response of chicks to different clinical isolates of avian adenovirus type-1. Comparative Immunology Microbiology and Infectious Diseases, 20: 59-62.

Mansoor, M.K., Hussain, I., Arshad, M. \& Muhammad, G. 2011. Preparation and evaluation of chicken embryo-adapted fowl adenovirus serotype 4 vaccine in broiler chickens. Tropical Animal Health and Production, 43: 331-338.
Marek, A., Kaján, G.L., Kosiol, C., Benkõ, M., Schachner, A. \& Hess, M. 2016. Genetic diversity of species Fowl aviadenovirus D and Fowl aviadenovirus E. Journal of General Virology, 97(9): 2323-2332.

Mat Isa, N., Mohd Ayob, J., Ravi, S., Mustapha, N.A., Ashari, K.S., Bejo, M.H., Omar, A.R. \& Ideris, A. 2019. Complete genome sequence of fowl adenovirus-8b UPM04217 associated with the inclusion body hepatitis disease in commercial broiler chickens in Malaysia reveals intermediate evolution. Virus Disease, 30: 426432.

Matthews, T.D. 2005. Protection of broiler breeders against a strain of fowl adenovirus and characterization of several fowl adenovirus serotypes (Master). University of Georgia, Athens.

Mirzazadeh, A., Asasi, K., Schachner, A., Mosleh, N., Liebhart, D., Hess, M. \& Grafl, B. 2019. Gizzard Erosion Associated with Fowl Adenovirus Infection in Slaughtered Broiler Chickens in Iran. Avian Diseases, 63(4): 568-576.

Morshed, R., Hosseini, H., Langeroudi, A.G., Fard, M.H.B. \& Charkhkar, S. 2017. Fowl adenoviruses $\mathrm{D}$ and $\mathrm{E}$ cause inclusion body hepatitis outbreaks in broiler and broiler breeder pullet flocks. Avian Diseases, 61(2): 205-210.

Norfitriah, M.S., Hair-Bejo, M., Omar, A.R., Aini, I. \& Nurulfiza, M.I. 2018. Molecular detection and pathogenicity of fowl adenovirus isolated from disease outbreak in commercial layer chickens. International Journal of Agricultural Sciences and Veterinary Medicine, 6(1): 73-84.

Norfitriah, M.S., Hair-Bejo, M. \& Majdi, A. 2019. Pathogenicity of fowl adenovirus serotype $8 \mathrm{~b}$ isolates of Malaysia in specific pathogen free chicken. Journal of Animal and Veterinary Advances, 18(3): 78-83.

Norina, L., Norsharina, A., Nurnadiah, A.H., Redzuan, I., Ardy, A. \& Nor-Ismaliza, A. 2016. Avian denovirus isolated broiler affected with inclusion body hepatitis. Malaysian Journal of Veterinary Research, 7(2): 121-126.

Rashid, F., Xie, Z., Zhang, L., Luan, Y., Luo, S., Deng, X., Xie, L., Xie, Z. \& Fan Q. 2020. Genetic characterization of fowl aviadenovirus 4 isolates from Guangxi, China, during 2017-2019. Poultry Science, 99(9): 4166-4173.

Reed, L.J. \& Muench, H. 1938. A simple method of estimating fifty per cent endpoints. American Journal of Hygiene, 27: 493-497.

Schachner, A., Matos, M., Grafl, B. \& Hess, M. 2018. Fowl adenovirus-induced diseases and strategies for their control - a review on the current global situation. Avian Pathology, 47(2): 111-126. 
Schijns, V.E.J.C., Zande, S.V.D, Lupiani, B. \& Reddy, S.M. (2014). Practical aspects of poultry vaccination. In: Avian Immunology, 2nd Ed. K.A. Schat, B. Kaspers, and P. Kaiser (Eds.). Academic Press, Elsevier Ltd., London, UK. pp. 345-362.

Schonewille, E., Jaspers, R., Paul, G. \& Hess, M. 2010. Specific-Pathogen - Free chickens vaccinated with a live FAdV-4 vaccine are fully protected against a severe challenge even in the absence of neutralizing antibodies. Avian Diseases, 54(2): 905-910.

Shah, M.S., Ashraf, A., Khan, M.I., Rahman, M., Habib, M., Chughtai, M.I. \& Qureshi, J.A. 2017. Fowl adenovirus: history, emergence, biology and development of a vaccine against hydropericardium syndrome. Archives of Virology, 162(7): 1833-1843.

Sohaimi, N.M., Bejo, M.H., Omar, A.R., Ideris, A. \& Isa, N.M. 2019. Molecular characterisation of fowl adenovirus isolate of Malaysia attenuated in chicken embryo liver cells and its pathogenicity and immunogenicity in chickens. PLoS $O N E$, 14(12): e0225863.

Steer, P.A., O'Rourke, D. Ghorashi, S.A. \& Noormohammadi, A.H. 2011. Application of high-resolution melting curve analysis for typing of fowl adenoviruses in field cases of inclusion body hepatitis. Australian Veterinary Journal, 89: 184-192.

Steer-Cope, P.A., Sandy, J.R., O’Rourke, D., Scott, P.C., Browning, G.F. \& Noormohammadi, A.H. 2019. Vaccination with FAdV-8a induces protection against inclusion body hepatitis caused by homologous and heterologous strains, Avian Pathology, 48(5): 396-405.
Wang, X., Tang, Q., Chu, Z., Wang, P., Luo, C., Zhang, Y., Fang, X., Qiu, L., Dang, R. \& Yang, Z. 2018. Immune protection efficacy of FAdV-4 surface proteins fiber-1, fiber-2, hexon and penton base. Virus Research, 245: 1-6.

Wang, J., Zaheer, I., Saleemi, M.K., Qi, X., Gao, Y., Cui, H., Li, K., Gao, L., Fayyaz, A., Hussain, A., Liu, C., Zhang, Y., Wang, X. \& Pan, Q. 2020. The first complete genome sequence and pathogenicity characterization of fowl adenovirus 11 from chickens with inclusion body hepatitis in Pakistan. Veterinary Microbiology, 244: 108670.

Wilcox, R.R. 1990. Comparing the means of two independent groups. Biometrical Journal, 32(7): 771-780.

Yasmeen, S., Siddique, N., Athar Abbas, M., Ali, A., Rafique, S., Rashid, F., Shah, A.U., Mehmood, F., Begum, I., Javaid, T., Jaffery, S., Ali, R. \& Naeem, K. 2017. Fiber gene based molecular and biological characterization of hydropericardiumhepatitis syndrome associated avian adenoviruses. Iranian Journal of Veterinary Research, 18(3): 190-196.

Zhang, Y., Liu, R., Tian, K., Wang, Z., Yang, X., Gao, D., Zhang, Y., Fu, J., Wang, H. \& Zhao, J. 2018. Fiber2 and hexon genes are closely associated with the virulence of the emerging and highly pathogenic fowl adenovirus 4. Emerging Microbes and Infections, 7(1): 199. 
